\title{
Hamiltonian chaos with a cold atom in an optical lattice
}

\author{
S.V. Prants
}

\begin{abstract}
We consider a basic model of the lossless interaction between a moving two-level atom and a standing-wave single-mode laser field. Classical treatment of the translational atomic motion provides the semiclassical Hamilton-Schrödinger equations of motion which are a five-dimensional nonlinear dynamical system with two integrals of motion. The atomic dynamics can be regular or chaotic (in the sense of exponential sensitivity to small variations in initial conditions and/or the system's control parameters) in dependence on values of the control parameters, the atom-field detuning and recoil frequency. We develop a semiclassical theory of the chaotic atomic transport in terms of a random walk of the atomic electric dipole moment $u$ which is one of the components of a Bloch vector. Based on a jump-like behavior of this variable for atoms crossing nodes of the standing laser wave, we construct a stochastic map that specifies the center-of-mass motion. We find the relations between the detuning, recoil frequency and the atomic energy, under which atoms may move in a rigid optical lattice in a chaotic way. We obtain the analytical conditions under which deterministic atomic transport has fractal properties and explain a hierarchical structure of the dynamical fractals. Quantum treatment of the atomic motion in a standing wave is studied in the dressed state picture where the atom moves in two optical potentials simultaneously. If the values of the detuning and a characteristic atomic frequency are of the same order, than there is a probability of nonadiabatic transitions of the atom upon crossing nodes of the standing wave. At the same condition exactly, we observe sudden changes (jumps) in the atomic dipole moment $u$ when the atom crosses the nodes. Those jumps are accompanied by splitting of atomic wave packets at the nodes. Such a proliferation of wave packets at the nodes of a standing wave is a manifestation

S.V. Prants

Laboratory of Nonlinear Dynamical Systems, Pacific Oceanological Institute of the Russian Academy of Sciences, 43 Baltiiskaya st., 690041 Vladivostok, Russia, e-mail: prants@poi.dvo.ru
\end{abstract}


of classical atomic chaotic transport. In particular, the effect of simultaneous trapping of an atom in a well of one of the optical potential and its flight in the other potential is a quantum analogue of a chaotic classical walking of an atom. At large values of the detuning, the quantum evolution is shown to be adiabatic in accordance with a regular character of the classical atomic motion.

\section{Short historical background}

The fundamental model for the interaction of a radiation with matter, comprising a collection of two-level quantum systems coupled with a single-mode electromagnetic field, provides the basis for laser physics and describes a rich variety of nonlinear dynamical effects. The discovery that a single-mode laser, a symbol of coherence and stability, may exhibit deterministic instabilities and chaos is especially important since lasers provide nearly ideal systems to test general ideas in statistical physics. From the stand point of nonlinear dynamics, laser is an open dissipative system which transforms an external excitation into a coherent output in the presence of loss. In 1975 Haken [1] has shown that a single-mode, homogeneously broadened laser, operating on resonance with the gain center can be described in the rotating-wave approximation by three real semiclassical Maxwell-Bloch equations which are isomorphic to the famous Lorenz equations. Some manifestations of a Lorenztype strange attractor and dissipative chaos have been observed with different types of lasers.

In the same time George Zaslavsky with co-workers 2 have studied interaction of an ensemble of two-level atoms with their own radiation field in a perfect single-mode cavity without any losses and external excitations, which is known as the Dicke model [3. They were able to demonstrate analytically and numerically dynamical instabilities and chaos of Hamiltonian type in a semiclassical version of the Dicke model without rotating-wave approximation. It was the first paper that opened the door to study Hamiltonian atomic chaos in the rapidly growing fields of cavity quantum electrodynamics, quantum and atomic optics. Semiclassical equations of motion for this system may be reduced to Maxwell-Bloch equations for three real independent variables which, in difference from the laser theory, do not include losses and pump. Those equations are, in general, nonintegrable, but they become integrable immediately after adopting the rotating-wave approximation 4 that implies the existence of an additional integral of motion, conservation of the so-called number of excitations. Numerical experiments have shown that prominent chaos arises when the density of atoms is very large (approximately $10^{20} \mathrm{~cm}^{3}$ in the optical range [2]). The following progress in this field has been motivated, mainly, by a desire to find manifestations of Hamiltonian atomic chaos in the models more suitable for experimental implementations. 
Twenty years after that pioneer paper, manifestations of Hamiltonian chaos have been found in experiments with kicked cold atoms in a modulated laser field. Nowdays, a few groups in the USA, Australia, New Zealand, Germany, France, England, Italy and in other countries can perform routine experiments on Hamiltonian chaos with cold atoms in optical lattices and traps (for a review see [12]).

In this paper we review some results on theory of Hamiltonian chaos with a single two-level atom in a standing-wave laser field that have been obtained in our group in Vladivostok. In spite of we published with George only one paper on this subject [6], our work in this field has been mainly inspired by his paper [2] written in 1975 in Krasnoyarsk, Siberia.

\section{Introduction}

An atom placed in a laser standing wave is acted upon by two radiation forces, deterministic dipole and stochastic dissipative ones 7 . The mechanical action of light upon neutral atoms is at the heart of laser cooling, trapping, and Bose-Einstein condensation. Numerous applications of the mechanical action of light include isotope separation, atomic lithography and epitaxy, atomicbeam deflection and splitting, manipulating translational and internal atomic states, measurement of atomic positions, and many others. Atoms and ions in an optical lattice, formed by a laser standing wave, are perspective objects for implementation of quantum information processing and quantum computing. Advances in cooling and trapping of atoms, tailoring optical potentials of a desired form and dimension (including one-dimensional optical lattices), controlling the level of dissipation and noise are now enabling the direct experiments with single atoms to study fundamental principles of quantum physics, quantum chaos, decoherence, and quantum-classical correspondence (for recent reviews on cold atoms in optical lattices see Ref. [8, 9]).

Experimental study of quantum chaos has been carried out with ultracold atoms in $\delta$-kicked optical lattices [10, 11, 12. To suppress spontaneous emission and provide a coherent quantum dynamics atoms in those experiments were detuned far from the optical resonance. Adiabatic elimination of the excited state amplitude leads to an effective Hamiltonian for the center-ofmass motion [13, whose $3 / 2$ degree-of-freedom classical analogue has a mixed phase space with regular islands embedded in a chaotic sea. De Brogile waves of $\delta$-kicked ultracold atoms have been shown to demonstrate under appropriate conditions the effect of dynamical localization in momentum distributions which means the quantum suppression of chaotic diffusion [10, 11, 12]. Decoherence due to spontaneous emission or noise tend to suppress this quantum effect and restore classical-like dynamics. Another important quantum chaotic phenomenon with cold atoms in far-detuned optical lattices is a chaosassisted tunneling. In experiments [14, 5] ultracold atoms have been demon- 
strated to oscillate coherently between two regular regions in mixed phase space even though the classical transport between these regions is forbidden by a constant of motion (other than energy).

The transport of cold atoms in optical lattices has been observed to take the form of ballistic motion, oscillations in wells of the optical potential, Brownian motion [15], anomalous diffusion and Lévy flights [16, 17]. The Lévy flights have been found in the context of subrecoil laser cooling [16] in the distributions of escape times for ultracold atoms trapped in the potential wells with momentum states close to the dark state. In those experiments the variance and the mean time for atoms to leave the trap have been shown to be infinite.

A new arena of quantum nonlinear dynamics with atoms in optical lattices is opened if we work near the optical resonance and take the dynamics of internal atomic states into account. A single atom in a standing-wave laser field may be semiclassically treated as a nonlinear dynamical system with coupled internal (electronic) and external (mechanical) degrees of freedom [18, 19, 20. In the semiclassical and Hamiltonian limits (when one treats atoms as pointlike particles and neglects spontaneous emission and other losses of energy), a number of nonlinear dynamical effects have been analytically and numerically demonstrated with this system: chaotic Rabi oscillations [18, 19, 20, Hamiltonian chaotic atomic transport and dynamical fractals [21, 22, 23, 25], Lévy flights and anomalous diffusion [6, 20, 24]. These effects are caused by local instability of the CM motion in a laser field. A set of atomic trajectories under certain conditions becomes exponentially sensitive to small variations in initial quantum internal and classical external states or/and in the control parameters, mainly, the atom-laser detuning. Hamiltonian evolution is a smooth process that is well described in a semiclassical approximation by the coupled Hamilton-Schrödinger equations. A detailed theory of Hamiltonian chaotic transport of atoms in a laser standing wave has been developed in our recent paper [23].

\section{Semiclassical dynamics}

\subsection{Hamilton-Schrödinger equations of motion}

We consider a two-level atom with mass $m_{a}$ and transition frequency $\omega_{a}$ in a one-dimensional classical standing laser wave with the frequency $\omega_{f}$ and the wave vector $k_{f}$. In the frame rotating with the frequency $\omega_{f}$, the Hamiltonian is the following:

$$
\hat{H}=\frac{\hat{P}^{2}}{2 m_{a}}+\frac{1}{2} \hbar\left(\omega_{a}-\omega_{f}\right) \hat{\sigma}_{z}-\hbar \Omega\left(\hat{\sigma}_{-}+\hat{\sigma}_{+}\right) \cos k_{f} \hat{X} .
$$


Here $\hat{\sigma}_{ \pm, z}$ are the Pauli operators which describe the transitions between lower, $|1\rangle$, and upper, $|2\rangle$, atomic states, $\Omega$ is a maximal value of the Rabi frequency. The laser wave is assumed to be strong enough, so we can treat the field classically. Position $\hat{X}$ and momentum $\hat{P}$ operators will be considered in section "Semiclassical dynamics" as $c$-numbers, $X$ and $P$. The simple wavefunction for the electronic degree of freedom is

$$
|\Psi(t)\rangle=a(t)|2\rangle+b(t)|1\rangle,
$$

where $a$ and $b$ are the complex-valued probability amplitudes to find the atom in the states $|2\rangle$ and $|1\rangle$, respectively. Using the Hamiltonian (11), we get the Schrödinger equation

$$
\begin{aligned}
i \frac{d a}{d t} & =\frac{\omega_{a}-\omega_{f}}{2} a-\Omega b \cos k_{f} X, \\
i \frac{d b}{d t} & =\frac{\omega_{f}-\omega_{a}}{2} b-\Omega a \cos k_{f} X .
\end{aligned}
$$

Let us introduce instead of the complex-valued probability amplitudes $a$ and $b$ the following real-valued variables:

$$
u \equiv 2 \operatorname{Re}\left(a b^{*}\right), \quad v \equiv-2 \operatorname{Im}\left(a b^{*}\right), \quad z \equiv|a|^{2}-|b|^{2},
$$

where $u$ and $v$ are a synchronized (with the laser field) and a quadrature components of the atomic electric dipole moment, respectively, and $z$ is the atomic population inversion.

In the process of emitting and absorbing photons, atoms not only change their internal electronic states but their external translational states change as well due to the photon recoil. In this section we will describe the translational atomic motion classically. The position and momentum of a point-like atom satisfy classical Hamilton equations of motion. Full dynamics in the absence of any losses is now governed by the Hamilton-Schrödinger equations for the real-valued atomic variables

$$
\begin{gathered}
\dot{x}=\omega_{r} p, \quad \dot{p}=-u \sin x, \quad \dot{u}=\Delta v, \\
\dot{v}=-\Delta u+2 z \cos x, \quad \dot{z}=-2 v \cos x,
\end{gathered}
$$

where $x \equiv k_{f} X$ and $p \equiv P / \hbar k_{f}$ are normalized atomic center-of-mass position and momentum, respectively. Dot denotes differentiation with respect to the dimensionless time $\tau \equiv \Omega t$. The normalized recoil frequency, $\omega_{r} \equiv \hbar k_{f}^{2} / m_{a} \Omega \ll 1$, and the atom-field detuning, $\Delta \equiv\left(\omega_{f}-\omega_{a}\right) / \Omega$, are the control parameters. The system has two integrals of motion, namely the total energy

$$
H \equiv \frac{\omega_{r}}{2} p^{2}-u \cos x-\frac{\Delta}{2} z
$$


and the Bloch vector $u^{2}+v^{2}+z^{2}=1$. The conservation of the Bloch vector length follows immediately from Eqs. (4).

Equations (5) constitute a nonlinear Hamiltonian autonomous system with two and half degrees of freedom which, owing to two integrals of motion, move on a three-dimensional hypersurface with a given energy value $H$. In general, motion in a three-dimensional phase space in characterized by a positive Lyapunov exponent $\lambda$, a negative exponent equal in magnitude to the positive one, and zero exponent. The maximum Lyapunov exponent characterizes the mean rate of the exponential divergence of initially close trajectories and serves as a quantitative measure of dynamical chaos in the system. The result of computation of the maximum Lyapunov exponent in dependence on the detuning $\Delta$ and the initial atomic momentum $p_{0}$ is shown in Fig. 1 Color in the plot codes the value of the maximum Lyapunov exponent $\lambda$. In white

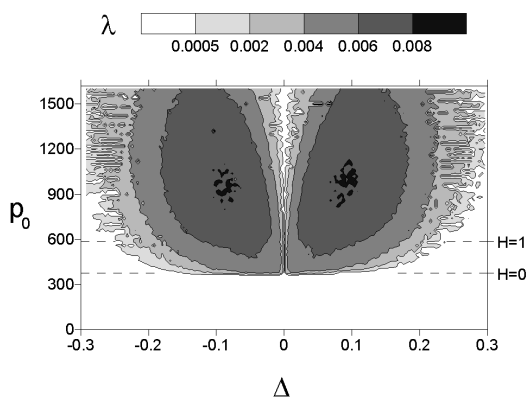

Fig. 1 Maximum Lyapunov exponent $\lambda$ vs atom-field detuning $\Delta$ and initial atomic momentum $p_{0}: \omega_{r}=10^{-5}, u_{0}=z_{0}=0.7071, v_{0}=0$.

regions the values of $\lambda$ are almost zero, and the atomic motion is regular in the corresponding ranges of $\Delta$ and $p_{0}$. In shadowed regions positive values of $\lambda$ imply unstable motion.

Figure 1 demonstrates that the center-of-mass motion becomes unstable if the dimensionless momentum exceeds the value $p_{0} \approx 300$ that corresponds (with our normalization) to the atomic velocity $v_{a} \approx 3 \mathrm{~m} / \mathrm{s}$ for an atom with $m_{a} \approx 10^{-22} \mathrm{~g}$ in the field with the wavelength close to the transition wavelength $\lambda_{a} \simeq 800 \mathrm{~nm}$. With these estimates for the atomic and lattice parameters and $\Omega / 2 \pi=10^{9}$, one gets the normalized value of the recoil frequency equal to $\omega_{r}=10^{-5}$. The detuning $\Delta$ will be varied in a wide range, and the Bloch variables are restricted by the length of the Bloch vector. 


\subsection{Regimes of motion}

The case of exact resonance, $\Delta=0$, was considered in detail in Ref. [18, 24]. Now we briefly repeat the simple results for the sake of self-consistency. At zero detuning, the variable $u$ becomes a constant, $u=u_{0}$, and the fast $(u, v$, $z$ ) and slow $(x, p)$ variables are separated allowing one to integrate exactly the reduced equations of motion. The total energy (6) is equal to $H_{0}=H(u=$ $\left.u_{0}, \Delta=0\right)$, and the atom moves in a simple cosine potential $u_{0} \cos x$ with three possible types of trajectories: oscillator-like motion in a potential well if $H_{0}<u_{0}$ (atoms are trapped by the standing-wave field), motion along the separatrix if $H_{0}=u_{0}$, and ballistic-like motion if $H_{0}>u_{0}$. The exact solution for the center-of-mass motion is easily found in terms of elliptic functions (see [18, 24]).

As to internal atomic evolution, it depends on the translational degree of freedom since the strength of the atom-field coupling depends on the position of atom in a periodic standing wave. At $\Delta=0$, it is easy to find the exact solutions of Eqs. (5)

$$
\begin{aligned}
& v(\tau)= \pm \sqrt{1-u^{2}} \cos \left(2 \int_{0}^{\tau} \cos x d \tau^{\prime}+\chi_{0}\right), \\
& z(\tau)=\mp \sqrt{1-u^{2}} \sin \left(2 \int_{0}^{\tau} \cos x d \tau^{\prime}+\chi_{0}\right),
\end{aligned}
$$

where $u=u_{0}$, and $\cos [x(\tau)]$ is a given function of the translational variables only which can be found with the help of the exact solution for $x$ [18, 24]. The sign of $v$ is equal to that for the initial value $z_{0}$ and $\chi_{0}$ is an integration constant. The internal energy of the atom, $z$, and its quadrature dipolemoment component $v$ could be considered as frequency-modulated signals with the instant frequency $2 \cos [x(\tau)]$ and the modulation frequency $\omega_{r} p(\tau)$, but it is correct only if the maximum value of the first frequency is much greater than the value of the second one, i. e., for $\left|\omega_{r} p_{0}\right| \ll 2$.

The maximum Lyapunov exponent $\lambda$ depends both on the parameters $\omega_{r}$ and $\Delta$, and on initial conditions of the system (5). It is naturally to expect that off the resonance atoms with comparatively small values of the initial momentum $p_{0}$ will be at once trapped in the first well of the optical potential, whereas those with large values of $p_{0}$ will fly through. The question is what will happen with atoms, if their initial kinetic energy will be close to the maximum of the optical potential. Numerical experiments demonstrate that such atoms will wander in the optical lattice with alternating trappings in the wells of the optical potential and flights over its hills. The direction of the center-of-mass motion of wandering atoms may change in a chaotic way (in the sense of exponential sensitivity to small variations in initial conditions). A typical chaotically wandering atomic trajectory is shown in Fig. 2 , 


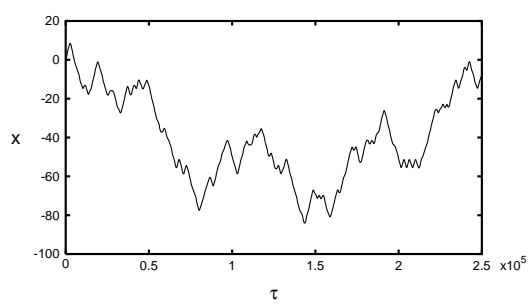

Fig. 2 Typical atomic trajectory in the regime of chaotic transport: $x_{0}=0, p_{0}=300$, $z_{0}=-1, u_{0}=v_{0}=0, \omega_{r}=10^{-5}, \Delta=-0.05$.

It follows from (5) that the translational motion of the atom at $\Delta \neq$ 0 is described by the equation of a nonlinear physical pendulum with the frequency modulation

$$
\ddot{x}+\omega_{r} u(\tau) \sin x=0,
$$

where $u$ is a function of all the other dynamical variables.

\subsection{Stochastic map for chaotic atomic transport}

Chaotic atomic transport occurs even if the normalized detuning is very small, $|\Delta| \ll 1$ (Fig. 11). Under this condition, we will derive in this section approximate equations for the center-of-mass motion. The atomic energy at $|\Delta| \ll 1$ is given with a good accuracy by its resonant value $H_{0}$. Returning to the basic set of the equations of motion (5), we may neglect the first right-hand term in the fourth equation since it is very small as compared with the second one there. However, we cannot now exclude the third equation from the consideration. Using the solution (7) for $v$, we can transform this equation as

$$
\dot{u}= \pm \Delta \sqrt{1-u^{2}} \cos \chi, \quad \chi \equiv 2 \int_{0}^{\tau} \cos x d \tau^{\prime}+\chi_{0} .
$$

Far from the nodes of the standing wave, Eq. (9) can be approximately integrated under the additional condition, $\left|\omega_{r} p\right| \ll 1$, which is valid for the ranges of the parameters and the initial atomic momentum where chaotic transport occurs. Assuming $\cos x$ to be a slowly-varying function in comparison with the function $\cos \chi$, we obtain far from the nodes the approximate solution for the $u$-component of the atomic dipole moment

$$
u \approx \sin \left( \pm \frac{\Delta}{2 \cos x} \sin \chi+C\right)
$$


where $C$ is an integration constant. Therefore, the amplitude of oscillations of the quantity $u$ for comparatively slow atoms $\left(\left|\omega_{r} p\right| \ll 1\right)$ is small and of the order of $|\Delta|$ far from the nodes.

At $|\Delta|=0$, the synchronized component of the atomic dipole moment $u$ is a constant whereas the other Bloch variables $z$ and $v$ oscillate in accordance with the solution (77). At $|\Delta| \neq 0$ and far from the nodes, the variable $u$ performs shallow oscillations for the natural frequency $|\Delta|$ is small as compared with the Rabi frequency. However, the behavior of $u$ is expected to be very special when an atom approaches to any node of the standing wave since near the node the oscillations of the atomic population inversion $z$ slow down and the corresponding driving frequency becomes close to the resonance with the natural frequency. As a result, sudden "jumps" of the variable $u$ are expected to occur near the nodes. This conjecture is supported by the numerical simulation. In Fig. 3 we show a typical behavior of the variable $u$ for a comparatively slow and slightly detuned atom. The plot clearly demon-

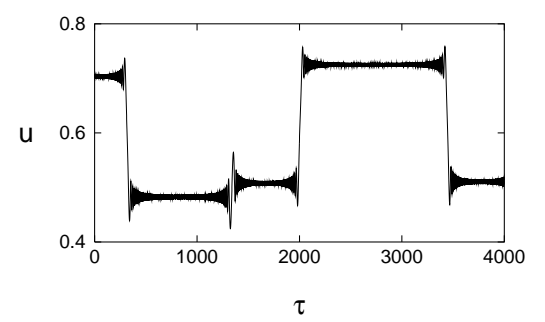

Fig. 3 Typical evolution of the atomic dipole-moment component $u$ for a comparatively slow and slightly detuned atom: $x_{0}=0, p_{0}=550, v_{0}=0, u_{0}=z_{0}=0.7071, \omega_{r}=10^{-5}$, $\Delta=-0.01$.

strates sudden "jumps" of $u$ near the nodes of the standing wave and small oscillations between the nodes.

Approximating the variable $u$ between the nodes by constant values, we can construct a discrete mapping [23]

$$
u_{m}=\sin \left(\Theta \sin \phi_{m}+\arcsin u_{m-1}\right),
$$

where $\Theta \equiv|\Delta| \sqrt{\pi / \omega_{r} p_{\text {node }}}$ will be called an angular amplitude of the jump, $u_{m}$ is a value of $u$ just after the $m$-th node crossing, $\phi_{m}$ are random phases to be chosen in the range $[0,2 \pi]$, and $p_{\text {node }} \equiv \sqrt{2 H / \omega_{r}}$ is the value of the atomic momentum at the instant when the atom crosses a node (which is the same with a given value of the energy $H$ for all the nodes). With given values of $\Delta, \omega_{r}$, and $p_{\text {node }}$, the map (11) has been shown numerically to give a satisfactory probabilistic distribution of magnitudes of changes in the variable $u$ just after crossing the nodes. The stochastic map (11) is valid under the assumptions of small detunings $(|\Delta| \ll 1)$ and comparatively slow 
atoms $\left(\left|\omega_{r} p\right| \ll 1\right)$. Furthermore, it is valid only for those ranges of the control parameters and initial conditions where the motion of the basic system (5) is unstable. For example, in those ranges where all the Lyapunov exponents are zero, $u$ becomes a quasi-periodic function and cannot be approximated by the map.

\subsection{Statistical properties of chaotic transport}

With given values of the control parameters and the energy $H$, the centerof-mass motion is determined by the values of $u_{m}$ (see Eq. (8) ). One can obtain from the expression for the energy (6) the conditions under which atoms continue to move in the same direction after crossing a node or change the direction of motion not reaching the nearest antinode. Moreover, as in the resonance case, there exist atomic trajectories along which atoms move to antinodes with the velocity going asymptotically to zero. It is a kind of separatrix-like motion with an infinite time of reaching the stationary points.

The conditions for different regimes of motion depend on whether the crossing number $m$ is even or odd. Motion in the same direction occurs at $(-1)^{m+1} u_{m}<H$, separatrix-like motion - at $(-1)^{m+1} u_{m}=H$, and turns at $(-1)^{m+1} u_{m}>H$. It is so because even values of $m$ correspond to $\cos x>0$, whereas odd values - to $\cos x<0$. The quantity $u$ during the motion changes its values in a random-like manner (see Fig. (3) taking the values which provide the atom either to prolong the motion in the same direction or to turn. Therefore, atoms may move chaotically in the optical lattice. The chaotic transport occurs if the atomic energy is in the range $0<H<1$. At $H<0$, atoms cannot reach even the nearest node and oscillate in the first potential well in a regular manner (see Fig. 1). At $H>1$, the values of $u$ are always satisfy to the flight condition. Since the atomic energy is positive in the regime of chaotic transport, the corresponding conditions can be summarized as follows: at $|u|<H$, atom always moves in the same direction, whereas at $|u|>H$, atom either moves in the same direction, or turns depending on the sign of $\cos x$ in a given interval of motion. In particular, if the modulus of $u$ is larger for a long time then the energy value, then the atom oscillates in a potential well crossing two times each of two neighbor nodes in the cycle.

The conditions stated above allow to find a direct correspondence between chaotic atomic transport in the optical lattice and stochastic dynamics of the Bloch variable $u$. It follows from Eq. (11) that the jump magnitude $u_{m}-u_{m-1}$ just after crossing the $m$-th node depends nonlinearly on the previous value $u_{m-1}$. For analyzing statistical properties of the chaotic atomic transport, it is more convenient to introduce the map for $\arcsin u_{m}$ [23]

$$
\theta_{m} \equiv \arcsin u_{m}=\Theta \sin \phi_{m}+\arcsin u_{m-1},
$$


where the jump magnitude does not depend on a current value of the variable. The map (12) visually looks as a random motion of the point along a circle of unit radius (Fig. 4). The vertical projection of this point is $u_{m}$. The value

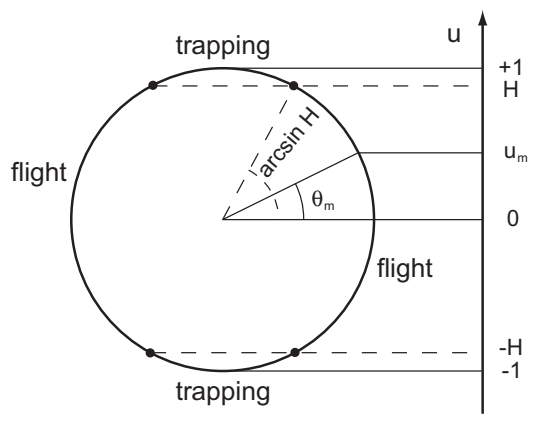

Fig. 4 Graphic representation for the maps of $u_{m}$ and $\theta_{m} \equiv \arcsin u_{m} . H$ is a given value of the atomic energy. Atoms either oscillate in optical potential wells (trapping) or fly through the optical lattice (flight).

of the energy $H$ specifies four regions, two of which correspond to atomic oscillations in a well, and two other ones - to ballistic motion in the optical lattice.

We will call "a flight" such an event when atom passes, at least, two successive antinodes (and three nodes). The continuous flight length $L>2 \pi$ is a distance between two successive turning points at which the atom changes the sign of its velocity, and the discrete flight length is a number of nodes $l$ the atom crossed. They are related in a simple way, $L \simeq \pi l$, for sufficiently long flight.

Center-of-mass oscillations in a well of the optical potential will be called "a trapping". At extremely small values of the detuning, the jump magnitudes are small and the trapping occurs, largely, in the $2 \pi$-wide wells, i. e., in the space interval of the length $2 \pi$. At intermediate values of the detuning, it occurs, largely, in the $\pi$-wide wells, i. e. in the space interval of the length $\pi$. Far from the resonance, $|\Delta| \gtrsim 1$, trapping occurs only in the $\pi$-wide wells. Just like to the case of flights, the number of nodes $l$, atom crossed being trapped in a well, is a discrete measure of trapping.

The PDFs for the flight $P_{\mathrm{fl}}(l)$ and trapping $P_{\mathrm{tr}}(l)$ events were analytically derived to be exponential in a case of large jumps [23. In a case of small jumps, the kind of the statistics depends on additional conditions imposed on the atomic and lattice parameters, and the distributions $P_{\mathrm{f}}(l)$ and $P_{\operatorname{tr}}(l)$ were analytically shown to be either practically exponential or functions with long power-law segments with the slope -1.5 but exponential "tails". The comparison of the PDFs computed with analytical formulas, the stochastic map, and the basic equations of motion has shown a good agreement in 
different ranges of the atomic and lattice parameters [23. We will use the results obtained to find the analytical conditions, under which the fractal properties of the chaotic atomic transport can be observed, and to explain the structure of the corresponding dynamical fractals.

Since the period and amplitude of the optical potential and the atom-field detuning can be modified in a controlled way, the transport exponents of the flight and trapping distributions are not fixed but can be varied continuously, allowing to explore different regimes of the atomic transport. Our analytical and numerical results with the idealized system have shown that deterministic atomic transport in an optical lattice cannot be just classified as normal and anomalous one. We have found that the flight and trapping PDFs may have long algebraically decaying segments and a short exponential "tail". It means that in some ranges of the atomic and lattice parameters numerical experiments reveal anomalous transport with Lévy flights. The transport exponent equal to -1.5 means that the first, second, and the other statistical moments are infinite for a reasonably long time. The corresponding atomic trajectories computed for this time are self-similar and fractal. The total distance, that the atom travels for the time when the flight PDF decays algebraically, is dominated by a single flight. However, the asymptotic behavior is close to normal transport. In other ranges of the atomic and lattice parameters, the transport is practically normal both for short and long times.

\subsection{Dynamical fractals}

Various fractal-like structures may arise in chaotic Hamiltonian systems 26, 27. In Ref. 22, 21, 24, 25] we have found numerically fractal properties of chaotic atomic transport in cavities and optical lattices. In this section we apply the analytical results of the theory of chaotic transport, developed in the preceding sections, to find the conditions under which the dynamical fractals may arise.

We place atoms one by one at the point $x_{0}=0$ with a fixed positive value of the momentum $p_{0}$ and compute the time $T$ when they cross one of the nodes at $x=-\pi / 2$ or $x=3 \pi / 2$. In these numerical experiments we change the value of the atom-field detuning $\Delta$ only. All the initial conditions $p_{0}=200$, $z_{0}=-1, u_{0}=v_{0}=0$ and the recoil frequency $\omega_{r}=10^{-5}$ are fixed. The exit

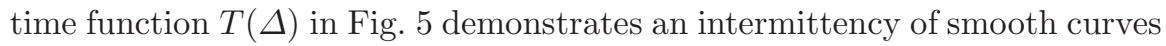
and complicated structures that cannot be resolved in principle, no matter how large the magnification factor. The second and third panels in Fig. 5 demonstrate successive magnifications of the detuning intervals shown in the upper panel. Further magnifications reveal a self-similar fractal-like structure that is typical for Hamiltonian systems with chaotic scattering [26, 28]. The exit time $T$, corresponding to both the smooth and unresolved $\Delta$ intervals, increases with increasing the magnification factor. Theoretically, there exist 


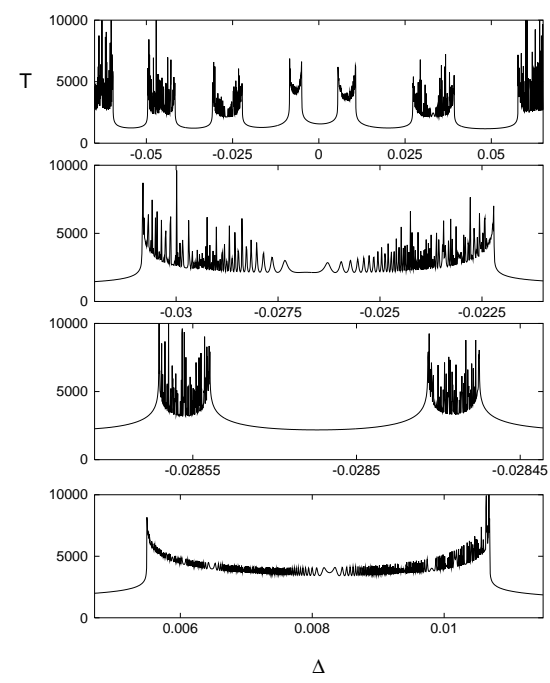

Fig. 5 Fractal-like dependence of the time of exit of atoms $T$ from a small region in the optical lattice on the detuning $\Delta: p_{0}=200, z_{0}=-1, u_{0}=v_{0}=0$. Magnifications of the detuning intervals are shown.

atoms never crossing the border nodes at $x=-\pi / 2$ or $x=3 \pi / 2$ in spite of the fact that they have no obvious energy restrictions to do that. Tiny interplay between chaotic external and internal atomic dynamics prevents those atoms from leaving the small space region.

Various kinds of atomic trajectories can be characterized by the number of times $m$ atom crosses the central node at $x=\pi / 2$ between the border nodes. There are also special separatrix-like trajectories along which atoms asymptotically reach the points with the maximum of the potential energy, having no more kinetic energy to overcome it. In difference from the separatrix motion in the resonant system $(\Delta=0)$, a detuned atom can asymptotically reach one of the stationary points even if it was trapped for a while in a well. Such an asymptotic motion takes an infinite time, so the atom will never reach the border nodes.

The smooth $\Delta$ intervals in the first-order structure (Fig. 5 , upper panel) correspond to atoms which never change the direction of motion $(m=1)$ and reach the border node at $x=3 \pi / 2$. The singular points in the first-order structure with $T=\infty$, which are located at the border between the smooth and unresolved $\Delta$ intervals, are generated by the asymptotic trajectories. Analogously, the smooth $\Delta$ intervals in the second-order structure (second panel in Fig. (5) correspond to the 2-nd order $(m=2)$ trajectories, and so on.

The set of all the values of the detunings, generating the separatrix-like trajectories, was shown to be a countable fractal in Refs. 21, 24, whereas the set of the values generating dynamically trapped atoms with $m=\infty$ seems 
to be uncountable. The exit time $T$ depends in a complicated way not only on the values of the control parameters but on initial conditions as well.

In Fig. 66 24] we presented a two-dimensional image of the time of exit $T$ in the space of the initial atomic momentum $p_{0}$ and the atom-field detuning $\Delta$. A self-similarity of this function is evident.
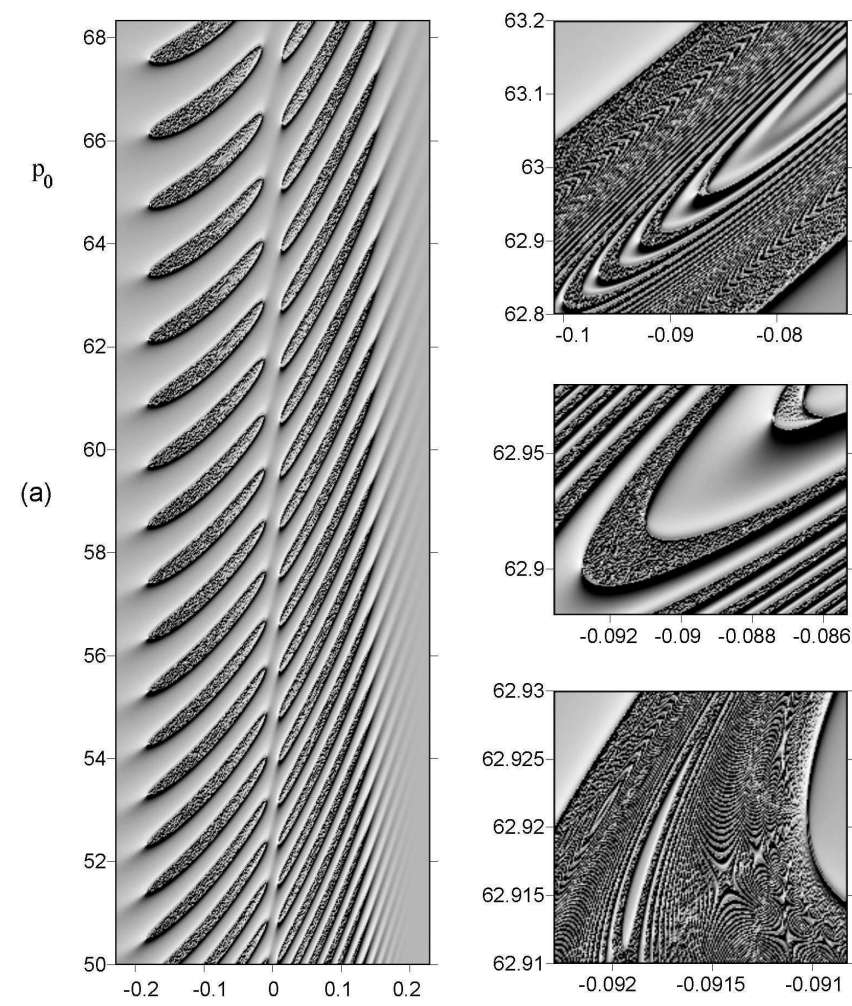

(c)

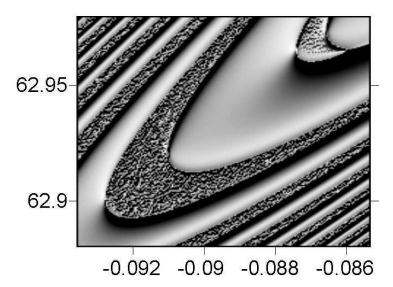

(d)

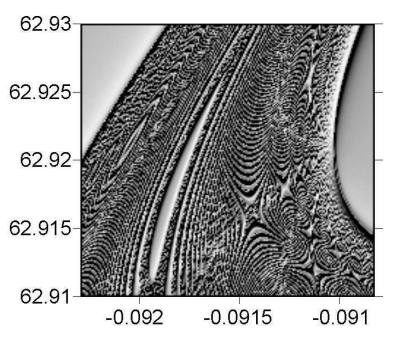

(e)
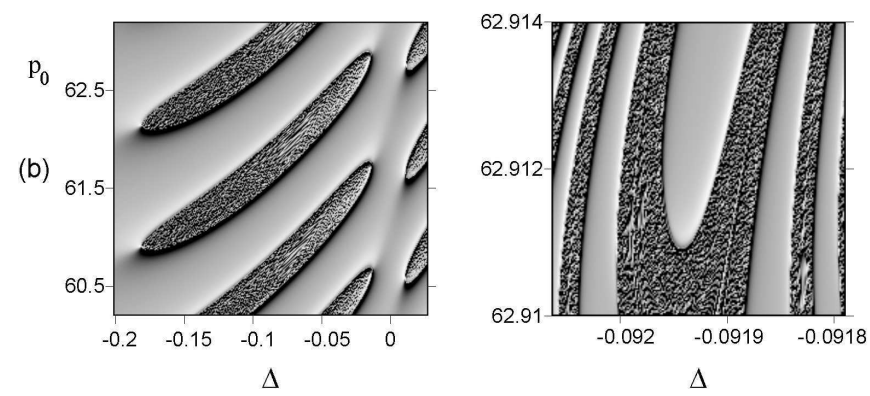

(f)

Fig. 6 The scattering function in the regime of chaotic wandering. The time of exit $T$ vs the detuning $\Delta$ and the initial momentum $p_{0}$. The function is shown in a shaded relief regime. 
The length of all smooth segments in the $m$-th order structure in Fig. [5] is proportional to the number of atoms $N(m)$ leaving the space $[-\pi / 2,3 \pi / 2]$ after crossing the central node $m$ times. An exponential scaling $N(m) \sim$ $\exp (-\gamma m)$ has been found numerically with $\gamma \simeq 1$. The trapping PDFs, computed with the basic and reduced equations of motion at the detunings in the range shown in Fig. 5, have been found to have exponential tails. It is well known [26] that Hamiltonian systems with fully developed chaos demonstrate, as a rule, exponential decay laws, whereas the systems with a mixed phase space (containing islands of regular motion) usually have more slow algebraic decays due to the effect of stickiness of trajectories to the boundaries of such islands [27. We have not found visible regular islands in our system at the values of the control parameters used to compute the fractal in Fig. 15 and we may conclude that the exponential scaling is a result of completely chaotic wandering of atoms in the space interval $[-\pi / 2,3 \pi / 2]$ resembling chaotic motion in hyperbolic systems.

The fractal-like structure with smooth and unresolved components may appear if atoms have an alternative either to turn back or to prolong the motion in the same direction just after crossing the node at $x=\pi / 2$. For the first-order structure in the upper panel in Fig. 5, it means that the internal variable $u$ of an atom, just after crossing the node for the first time $\left(\cos x<0\right.$ ), satisfies either to the condition $u_{1}<H$ (atom moves in the same direction), or to the condition $u_{1}>H$ (atom turns back). If $u_{1}=H$, then the exit time $T$ is infinite. The jumps of the variable $u$ after crossing the node are deterministic but sensitively dependent on the values of the control parameters and initial conditions. We have used this fact when introducing the stochastic map. Small variations in these values lead to oscillations of the quantity $\arcsin u_{1}$ around the initial value $\arcsin u_{0}$ with the angular amplitude $\Theta$. If this amplitude is large enough, then the sign of the quantity $u_{1}-H$ alternates and we obtain alternating smooth (atoms reach the border $x=3 \pi / 2$ without changing their direction of motion) and unresolved (atoms turns a number of times before exit) components of the fractal-like structure.

If the values of the parameters admit large jump magnitudes of the variable $u$, then the dynamical fractal arises in the energy range $0<H<1$, i. e., at the same condition under which atoms move in the optical lattice in a chaotic way. In a case of small jump magnitudes, fractals may arise if the initial value of an atom $u_{0}$ is close enough to the value of the energy $H$, i. e., the atom has a possibility to overcome the value $u=H$ in a single jump. Therefore, the condition for appearing in the fractal $T(\Delta)$ the first-order structure with singularities is the following:

$$
\left|\arcsin u_{0}-\arcsin H\right|<\Theta .
$$

The generation of the second-order structure is explained analogously. If an atom made a turn after crossing the node for the first time, then it will cross the node for the second time. After that, the atom either will turn or 
cross the border node at $x=-\pi / 2$. What will happen depend on the value of $u_{2}$. However, in difference from the case with $m=1$, the condition for appearing an infinite exit time with $m=2$ is $u_{2}=-H$. Furthermore, the previous value $u_{1}$ is not fixed (in difference from $u_{0}$ ) but depends on the value of the detuning $\Delta$. In any case we have $u_{1}>H$ since the second-order structure consists of the trajectories of those atoms which turned after the first node crossing. In order for an atom would be able to turn after the second node crossing, the magnitude of its variable $u$ should change sufficiently to be in the range $u_{2}<-H$. The atoms, whose variables $u$ could not "jump" so far, leave the space $[-\pi / 2,3 \pi / 2]$. The singularities are absent in the middle segment of the second-order structure shown in the second panel in Fig. 5 because all the corresponding atoms left the space after the second node crossing. The variable $u_{2}$ oscillates with varying $\Delta$ generating oscillations of the exit time. The condition for appearing singularities in the second-order structure is the following:

$$
2 \arcsin H<\Theta
$$

With the values of the parameters taken in the simulation, we get the energy $H=0.2+\Delta / 2$. It is easy to obtain from the inequality (14) the approximate value of the detuning $|\Delta| \approx 0.0107$ for which the second-order singularities

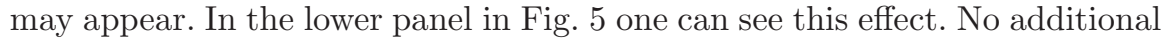
conditions are required for generating the structures of the third and the next orders.

Inequality (14) is opposite to the inequality that determines the condition for appearing power law decays in the flight PDF. Therefore, dynamical fractal may appear in those ranges of the control parameters where the Lévy flights are impossible and vice versa. However, the trapping PDF may have a power law decay. Inequality (14) in difference from (13) is strongly related

with the chosen concrete scheme for computing exit times. It is not required with other schemes, say, with three antinodes between the border nodes.

\section{Quantum dynamics}

In this section we will treat atomic translational motion quantum mechanically, i. e., atom is supposed to be not a point particle but a wave packet. The corresponding Hamiltonian $\hat{H}$ has the form (1) with $\hat{X}$ and $\hat{P}$ being the position and momentum operators. We will work in the momentum space with the state vector

$$
|\Psi(t)\rangle=\int(a(P, t)|2\rangle+b(P, t)|1\rangle)|P\rangle d P,
$$

which satisfies to the Schrödinger equation 


$$
i \hbar \frac{d|\Psi\rangle}{d t}=\hat{H}|\Psi\rangle
$$

The normalized equations for the probability amplitudes have the form

$$
\begin{aligned}
i \dot{a}(p) & =\frac{1}{2}\left(\omega_{r} p^{2}-\Delta\right) a(p)-\frac{1}{2}[b(p+1)+b(p-1)], \\
i \dot{b}(p) & =\frac{1}{2}\left(\omega_{r} p^{2}+\Delta\right) b(p)-\frac{1}{2}[a(p+1)+a(p-1)],
\end{aligned}
$$

with the same normalization and the control parameters as in the semiclassical theory. When deriving (17), we used the following property of the momentum operator $\hat{P}$ :

$$
\cos k_{f} \hat{X}|P\rangle \equiv \frac{1}{2}\left(e^{i k_{f} \hat{X}}+e^{-i k_{f} \hat{X}}\right)|P\rangle=\frac{1}{2}\left(\left|P+\hbar k_{f}\right\rangle+\left|P-\hbar k_{f}\right\rangle\right) .
$$

Equations (17) are an infinite-dimensional set of ordinary differential complexvalued equations of the first order with coupled amplitudes $a(p \pm n)$ and $b(p \pm m)$. To characterize the internal atomic state, let us introduce the following variables;

$$
\begin{aligned}
u(\tau) & \equiv 2 \operatorname{Re} \int d x\left[a(x, \tau) b^{*}(x, \tau)\right], \\
v(\tau) & \equiv-2 \operatorname{Im} \int d x\left[a(x, \tau) b^{*}(x, \tau)\right], \\
z(\tau) & \equiv \int d x\left[|a(x, \tau)|^{2}-|b(x, \tau)|^{2}\right],
\end{aligned}
$$

which are quantum versions of the Bloch components (44), and we denote them by the same letters.

\section{Dressed states picture and nonadiabatic transitions}

Interpretation of the atomic wave-packet motion in a standing-wave field is greatly facilitated in the basis of atomic dressed states which are eigenstates of a two-level atom in a laser field. The adiabatic dressed states

$$
\begin{gathered}
|+\rangle_{\Delta}=\sin \Theta|2\rangle+\cos \Theta|1\rangle, \quad|-\rangle_{\Delta}=\cos \Theta|2\rangle-\sin \Theta|1\rangle, \\
\tan \Theta \equiv \frac{\Delta}{2 \cos x}-\sqrt{\left(\frac{\Delta}{2 \cos x}\right)^{2}+1}
\end{gathered}
$$

are eigenstates at a nonzero detuning. The corresponding values of the quasienergy are 


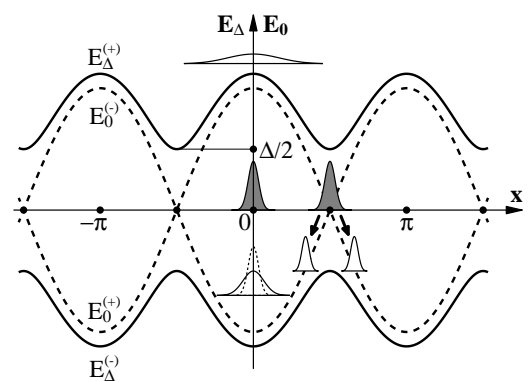

Fig. 7 Resonant $E_{0}^{( \pm)}$and nonresonant $E_{\Delta}^{( \pm)}$potentials for an atom in a standing wave. The optical Stern-Gerlach effect in the resonant potential is shown: splitting of an atomic wave packet launched at the node of the wave $\left(x_{0}=\pi / 2, p_{0}=0\right)$. The wave packet, placed initially at the antinode $\left(x_{0}=0, p_{0}=0\right)$, appears to be simultaneously at the top of $E_{0}^{(+)}$ and the bottom of $E_{0}^{(-)}$potentials. Its $|+\rangle$-component slides down both the sides of $E_{0}^{(+)}$ and the $|-\rangle$-component oscillates at the bottom of $E_{0}^{(-)}$.

$$
E_{\Delta}^{( \pm)}= \pm \sqrt{\frac{\Delta^{2}}{2}+\cos ^{2} x}
$$

Figure 7 shows a spatial variation of the quasienergies along the standingwave axis. It follows from Eqs.(20) and (21) that, in general case, atom moves in the two potentials $E_{\Delta}^{( \pm)}$simultaneously.

At exact resonance, $\Delta=0$, the dressed states have the simple form

$$
|+\rangle=\frac{1}{\sqrt{2}}(|1\rangle+|2\rangle), \quad|-\rangle=\frac{1}{\sqrt{2}}(|1\rangle-|2\rangle)
$$

and are called diabatic states. The resonant potentials, $E_{0}^{( \pm)}= \pm \cos x$, cross each other at the nodes of the standing wave, $x=\pi / 2+\pi m,(m=0, \pm 1, \ldots)$. What will happen if we place the centroid of an atomic wave packet exactly at the node, $x_{0}=\pi / 2$, in the ground state $|1\rangle$ and suppose its initial mean momentum to be zero, $p_{0}=0$ ? The initial ground state is the superposition of the diabatic states: $|1\rangle=(|+\rangle+|-\rangle) / \sqrt{2}$. One part of the initial wave packet at the top of the potential $E_{0}^{(+)}$will start to move to the right under the action of the gradient force $F^{(+)}=-d E_{0}^{(+)} / d x=\sin x$, and another one - to the left to be forced by $F^{(-)}=-\sin x$ (see Fig. 17). It is the wellknown optical Stern-Gerlach effect [29, 7, 30. If the maximal expected value of the atomic kinetic energy does not exceed the potential one, the atom will be trapped in the potential well. Two splitted components of the initial wave packet will oscillate in the well with the period of oscillations

$$
T \simeq 4 \sqrt{\frac{\pi}{\omega_{r}}} .
$$


The wave packet, with $p_{0}=0$, placed at the antinode, say, at $x_{0}=0$, is simultaneously at the top of the potential $E_{0}^{(+)}$and at the bottom of $E_{0}^{(-)}$. Therefore, its $|+\rangle$-component will slide down the both sides of the potential curve $E_{0}^{(+)}$, and the $|-\rangle$-component will oscillate around $x=0$ (see Fig. 77).

Out off resonance, $\Delta \neq 0$, the atomic wave packet moves in the bipotential $E_{\Delta}^{( \pm)}$(21). The distance between the quasienergy curves is minimal at the nodes of the standing wave and equal to $\Delta$ (see Fig. 7). The spatial period and the modulation depth of the resonant potentials $E_{0}^{( \pm)}$are twice as much as those for the nonresonant potentials $E_{\Delta}^{( \pm)}$.

The probability of nonadiabatic transitions between the dressed states $|+\rangle_{\Delta}$ and $|-\rangle_{\Delta}$ can be estimated in a simple way. The time of flight over a short distance $\delta x$ in neighbourhood of a node is $\delta x / \omega_{r} p_{\text {node }}$. If the time of transition between the quasienergy levels, $2 / \Delta$, is of the order of the flight time, the transition probability is close to 1 . It is easy to get the characteristic frequency of atomic motion from that condition [7]

$$
\Delta_{0}=\sqrt{\omega_{r} p_{\text {node }}}
$$

where $p_{\text {node }}$ is a value of the momentum in the vicinity of a node.

Depending on the relation between $\Delta$ and $\Delta_{0}$, there are three typical cases.

1. If $|\Delta| \ll \Delta_{0}$, the nonadiabatic transition probability between the states $|+\rangle_{\Delta}$ and $|-\rangle_{\Delta}$ upon crossing any node is close to 1 . However, the diabatic states $|+\rangle$ and $|-\rangle$ are not mixed, and atom moves in one of optical resonant potentials.

2. If $|\Delta| \simeq \Delta_{0}$, the atom may or may not undergo a transition upon crossing any node from one of the nonresonant potentials to another one with the probabilities of the same order.

3. If $|\Delta| \gg \Delta_{0}$, the nonadiabatic transition probability is exponentially small, and atom moves in one of the nonresonant potentials.

\subsection{Wave packet motion in the momentum space}

The atom at $\tau=0$ is supposed to be prepared as a Gaussian wave packet in the momentum space

$$
a_{0}(p)=0, \quad b_{0}(p)=\frac{1}{\sqrt{\sqrt{\pi} \Delta p}} \exp \left[-\frac{\left(p-p_{0}\right)^{2}}{2(\Delta p)^{2}}-i\left(p-p_{0}\right) x_{0}\right],
$$

with the momentum width $\Delta p=10$ corresponding to the spatial width $\Delta X=$ $\lambda_{f} / 40 \pi$ that is much smaller than the optical wavelength $\lambda_{f}$. We compute the probability to find a two-level atom at the moment of time $\tau$ with the momentum $p$ 


$$
W(p, \tau)=|a(p, \tau)|^{2}+|b(p, \tau)|^{2},
$$

by integrating Eqs. (17) with the initial condition (25). The recoil frequency, $\omega_{r}=10^{-5}$, is fixed and the centroid of the wave packet is placed at the antinode $x_{0}=0$, in all the numerical experiments.

\subsubsection{Adiabatic evolution at exact resonance}

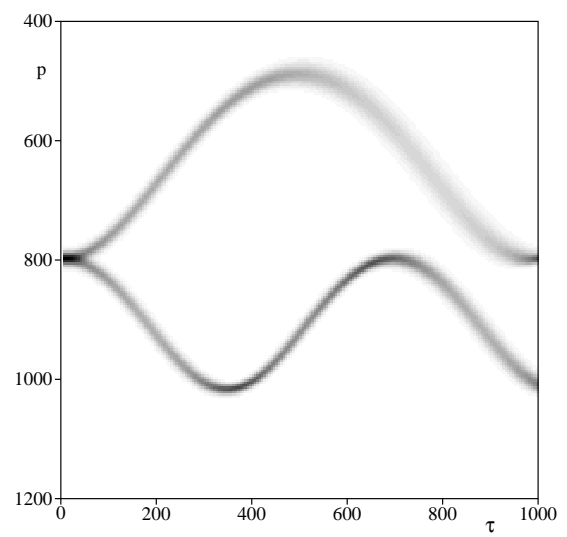

Fig. 8 Time dependence of the momentum probability function $W(p, \tau)$ for a ballistic atom at resonance prepared initially in the ground state $\left(\Delta=0, \omega_{r}=10^{-5}, x_{0}=0\right.$, $\left.p_{0}=800\right)$.

At exact resonance, $\Delta=0$, the wave functions of the diabatic states $|+\rangle$ and $|-\rangle$ evolve independently, each one evolves in its own potential $E_{0}^{(+)}$and $E_{0}^{(-)}$, respectively. The atom, prepared initially in the ground state $|1\rangle=(|+\rangle+|-\rangle) / \sqrt{2}$ with the mean initial momentum $p_{0}=800$, will start to move from the top of $E_{0}^{(+)}$and the bottom of $E_{0}^{(-)}$potentials (see Fig 7 ). Thus, the initial wave packet will split into two components $|+\rangle$ and $|-\rangle$. Time evolution of the probability function (26) for each of the components is shown in Fig 8 Pay, please, attention that the values of $p$ on this and similar plots increase downwards. Color in this figure codes the values of $W(p, \tau)$. The $|+\rangle$-component (the lower trajectory in the figure) slides down the curve $E_{0}^{(+)}$and, therefore, moves with an increasing velocity up to the next antinode at $x=\pi$, and then it slows down approaching the antinode at $x=2 \pi$. The atom moves in the positive direction of the axis $x$ and the process repeats periodically with the period $\tau_{0}^{(+)}=2 \pi / \omega_{r} \bar{p}_{0,2 \pi}^{(+)} \simeq 690$, where $\bar{p}_{0,2 \pi}^{(+)}$is a mean momentum of the $|+\rangle$-component upon the atomic motion between 0 and $2 \pi$. 
The $|-\rangle$-component (the upper trajectory in Fig 8 moves upward the potential curve $E_{0}^{(-)}$and slows down up to reaching the top of $E_{0}^{(-)}$at $x=\pi$. Then it moves with an increasing momentum up to $x=2 \pi$. Since the mean momentum of the $|-\rangle$-component is smaller than that of the $|+\rangle$ one, the corresponding period is longer, $\tau_{0}^{(-)} \simeq 980$.

\subsubsection{Proliferation of wave packets at the nodes of the standing wave}

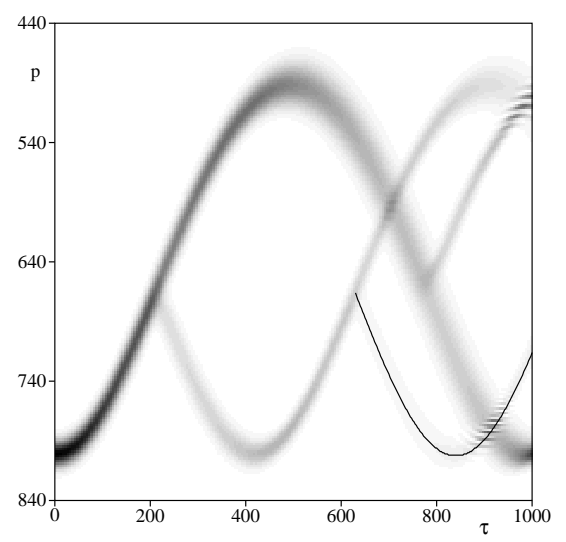

Fig. 9 Proliferation of atomic wave packets at the nodes of the standing wave at the detuning $\Delta=0.05$. The atom is prepared initially in the dressed state $|+\rangle$. Other conditions are the same as in Fig 8

New features in propagation of atomic wave packets through the standing wave appear under the condition $\Delta \simeq \Delta_{0}$. Using the semiclassical expression for the total atomic energy (6), let us estimate the value of the atomic momentum at the nodes of the standing wave if the detuning is not large, $|\Delta| \ll 1$. If the atom is prepared initially in the state $|+\rangle$, i.e., $u_{0}=1$, $z_{0}=0$, and $x_{0}=0$ then we have $H=H_{0}=2.2$ at $p_{0}=800$. Since the total energy is a constant, we get immediately from Eq. (6)

$$
p_{\text {node }} \simeq \sqrt{2 H / \omega_{r}} \simeq 665 .
$$

Using the same formula (6), we get the values of the minimal and maximal momenta if the atom starts to move with the initial mean momentum $p_{0}=$ 800: $p_{\min } \simeq \sqrt{2\left(H_{0}-1\right) / \omega_{r}} \simeq 490$ and $p_{\max } \simeq \sqrt{2\left(H_{0}+1\right) / \omega_{r}} \simeq 800$.

The formula (24) gives us the value of the characteristic frequency under the chosen conditions, $\Delta_{0} \simeq 0.08$. We fix $\Delta=0.05$ in this section, so $\Delta \simeq \Delta_{0}$. 
The initial state $|+\rangle$ is the following superposition of the adiabatic states:

$$
|+\rangle=\frac{1}{\sqrt{2}}\left[(\cos \Theta+\sin \Theta)|+\rangle_{\Delta}+(\cos \Theta-\sin \Theta)|-\rangle_{\Delta}\right] .
$$

With the help of (21) we can estimate the mixing angle at $\Delta=0.05$ to be equal to $\theta \simeq-\pi / 4$. Then it follows from (28) that $|+\rangle \simeq|-\rangle_{\Delta}$, i. e., practically all the wave packet is initially at the bottom of the potential $E_{\Delta}^{(-)}$ (Fig. 7). Figure 9 demonstrates that the wave packet really slows down, and its centroid intersects the node $x=\pi / 2$ at $\tau_{1}^{(-)} \simeq 215$. Under the condition $\Delta \simeq \Delta_{0}$, the atom has a probability to change the potential for another one upon crossing a node and a probability to stay in its present potential. This is exactly what we see in fig. 9 the wave packet splits at the node $x=\pi / 2$ with the $|-\rangle$-component climbing over the potential $E_{\Delta}^{(-)}$(see the upper trajectory in this figure) and the $|+\rangle$-component sliding down the curve $E_{\Delta}^{(+)}$with an increasing momentum (see the lower trajectory). Just after crossing the node, the most part of the probability density moves in the potential $E_{\Delta}^{(-)}$because the corresponding probability is larger. The $|+\rangle$component increases its velocity upon approaching the antinode at $x=\pi$ and then slows down up to the second node at $x=3 \pi / 2$ where it splits into two components at $\tau_{2}^{(+)} \simeq 640$. After that, one of the components will move in the potential $E_{\Delta}^{(+)}$decreasing the velocity up to the next antinode at $x=2 \pi$, and the other one will move in $E_{\Delta}^{(-)}$increasing its velocity in the same space interval. The probability density of this $|-\rangle$-component is only a few percents, and we draw a solid curve along this trajectory in order to visualize the motion.

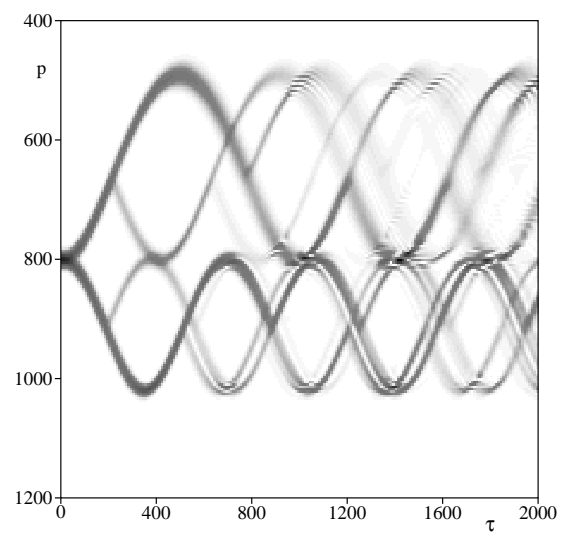

Fig. 10 The same as in Fig 9 but for the atom prepared initially in the ground state. 
The $|-\rangle$-component of the packet, splitted after crossing the first node at $x=\pi / 2$, has a smaller mean momentum than the $|+\rangle$-one. Therefore, it reaches the second node later, at $\tau_{2}^{(-)} \simeq 800$, where it splits into two parts: the upper $|+\rangle$-component will move in the potential $E_{\Delta}^{(+)}$and the lower $|-\rangle$-one - in $E_{\Delta}^{(-)}$. Such a proliferation of atomic wave packets takes places upon crossing all the next nodes of the standing wave.

The moment of time $\tau_{n}^{( \pm)}$, when the centroids of the $| \pm\rangle$-components cross the $n$-th node, can be estimated by the simple formula (we suppose that the centroid of the atomic wave packet was at $x=0$ at $\tau=0$ ):

$$
\omega_{r} \bar{p}_{n-1, n}^{( \pm)} \tau_{n}^{( \pm)}=(2 n-1) \frac{\pi}{2}, \quad n=2,3, \ldots
$$

where $\bar{p}_{n-1, n}^{( \pm)}$is a mean momentum of the $| \pm\rangle$-components upon their movement between $(n-1)$-th and $n$-th nodes. This quantity for the $|-\rangle$ component, moving between $x=0$ and $x=\pi / 2$, is $\bar{p}_{0,1}^{(-)}=\left(p_{0}+p_{\text {node }}\right) / 2 \simeq$ 732.5. So, the centroid of this wave packet crosses the first node at $\tau_{1}^{(-)} \simeq 214$. The lower $|+\rangle$-component crosses the second node at $x=3 \pi / 2$ at $\tau_{2}^{(+)} \simeq$ 642. For the upper $|-\rangle$-component we get $\bar{p}_{1,2}^{(-)}=\left(p_{\text {node }}+p_{\text {min }}\right) / 2 \simeq 577.5$ and $\tau_{2}^{(-)} \simeq 815$. All the other moments of time, $\tau_{n}^{( \pm)}$, can be estimated in the same way. The estimates obtained fit well the numerical data (see Fig 9). The interference fringes on the upper trajectory at $\tau \simeq 1000$ and $p \simeq 500$ and on the lower one at $\tau \simeq 900$ and $p \simeq 800$ reflect the fine-scale splitting of the corresponding wave packets.

Let us now compute the probability map for the atom prepared initially in the ground state $|1\rangle$ which has the following form in the adiabatic state basis:

$$
|1\rangle=\cos \Theta|+\rangle_{\Delta}-\sin \Theta|-\rangle_{\Delta},
$$

It follows from (21) that (30) is almost a $50 \%-50 \%$ superposition of the $|+\rangle_{\Delta}$ and $|-\rangle_{\Delta}$ states. All the other conditions are assumed to be the same as before. The atomic wave packet splits from the beginning into two components with the $|+\rangle$-one sliding down the curve $E_{\Delta}^{(+)}$(the lower trajectory in Fig. 10) and the $|-\rangle$-one climbing over the potential $E_{\Delta}^{(-)}$(the upper trajectory). Each of the components splits at the first node with a small time difference between the events. The subsequent proliferation of the wave packets occurs for the upper and lower parts of the probability density independently on each other in accordance with the same scenario as described above. In difference from the preceding case, the atom, prepared initially in the ground state, acquired the values of the momentum that are larger then the initial momentum $p_{0}=800$.

The nonadiabatic transitions are accompanied by drastic changes in the internal state of the atom which is characterized by the values of the synphased component of the electric dipole moment $u$ and the population inversion $z$. 


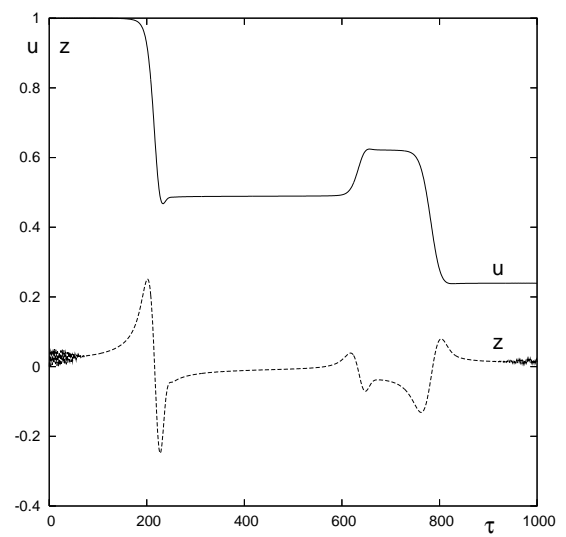

Fig. 11 Time dependence of the dipole moment $u$ and the population inversion $z$ at the same conditions as in Fig. 9

In Fig. 11 we demonstrate their behavior for the atom prepared initially in the state $|+\rangle$. Both the variables change their values abruptly in the time intervals with the centers at $\tau \simeq 215,640$ and 815 , i. e., when the centroids of the atomic wave packets cross the first two nodes.

\subsubsection{Adiabatic motion at large detunings}

For comparison with the results of the preceding section, we demonstrate in Fig. 12 the evolution of the momentum distribution function $W(p, \tau)$ with the ground initial state at $\Delta=2$ and the other same conditions as in the preceding section. The detuning $\Delta=2$ is large as compared to the characteristic frequency $\Delta_{0} \simeq 0.09$ that is estimated from (24) at $p_{0}=800$. It follows from (20) and (21) that at $\Delta=2$ the initial state $|1\rangle$ is a superposition of approximately $70 \%$ of the state $|+\rangle_{\Delta}$ and $\sim 30 \%$ of the state $|-\rangle_{\Delta}$. So the main part of the initial packet begins to move in the potential $E_{\Delta}^{(+)}$increasing the momentum upon approaching the node at $x=\pi / 2$, and the other part moves in $E_{\Delta}^{(-)}$decreasing the momentum in the same space interval (see Fig. 12). Upon crossing the nodes, the probability of transition between the states $| \pm\rangle_{\Delta}$ is small if $|\Delta| \gg \Delta_{0}$, and each of the component will continue to move in its own potential. The process is repeated and we see the periodic variations of the mean momentum of each of the components. The same picture is observed if we take the state $|+\rangle=(|1\rangle+|2\rangle) / \sqrt{2}$ as the initial one. At $\Delta=2$, the state $|+\rangle$ is a mix of $70 \%$ of $|-\rangle_{\Delta}$ and $30 \%$ of $|+\rangle_{\Delta}$, so the main part of the initial $|+\rangle$ wave packet will move in the potential $E_{\Delta}^{(-)}$. The evolution of the internal atomic variables $z$ and $u$ is shown in Fig. 13. There 
are no jumps of $z$ and $u$ when the atom crosses nodes. Instead of that, we see fast oscillations of those variables when the atom crosses the first antinodes.

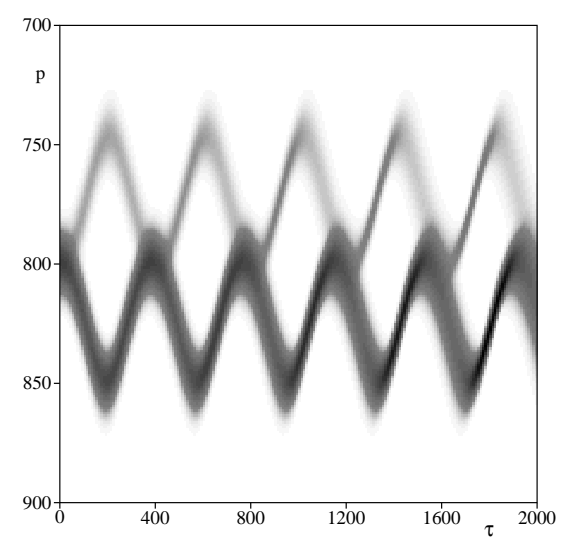

Fig. 12 Adiabatic evolution of the momentum probability function $W(p, \tau)$ for a ballistic atom at the large detuning $\Delta=2$.
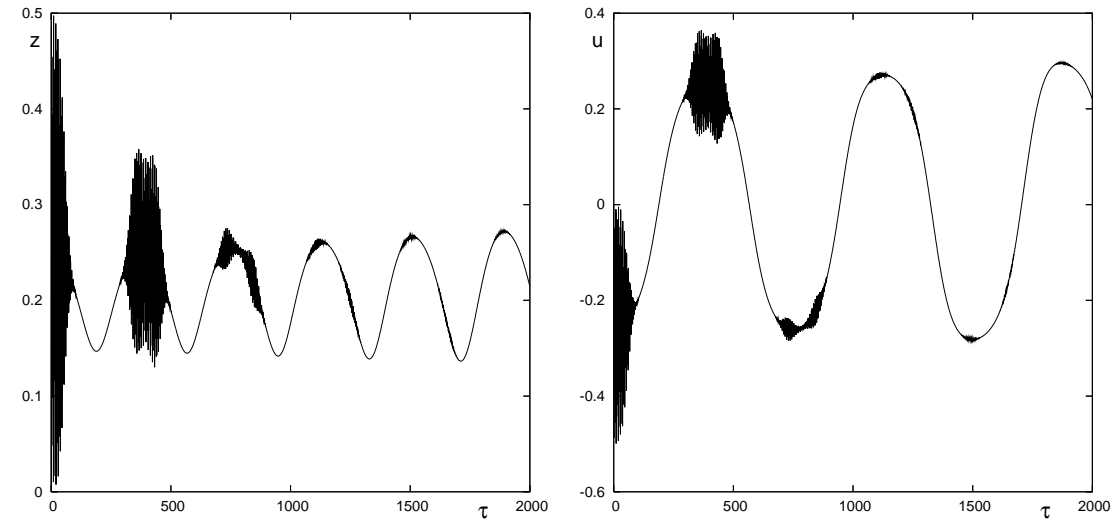

Fig. 13 The same as in Fig 11 but at the large detuning $\Delta=2$.

Thus, at $|\Delta| \gg \Delta_{0}$, there are no nonadiabatic transitions due to the corresponding small probability and, therefore, no proliferation of wave packets at the nodes. The evolution of the atomic wave packet is adiabatic. 


\subsubsection{An atom can fly and be trapped simultaneously}

An intriguing effect of simultaneous trapping of an atom in a well of the optical potential and its ballistic flight through the optical lattice is observed at comparatively small values of the detuning. Let us prepare an atom in the ground state $|1\rangle$ with such a mean initial value of the momentum $p_{0}$ that its $|-\rangle$-component would not be able to overcome the barrier of the potential $E_{\Delta}^{(-)}$but its $|+\rangle$-component would have a sufficient kinetic energy to overcome the barrier of the $E_{\Delta}^{(+)}$potential. Now one could expect periodic oscillations in the first well of the potential $E_{\Delta}^{(-)}$and a simultaneous ballistic flight in the $E_{\Delta}^{(+)}$potential with a proliferation of wave packets of the $|+\rangle$component at the nodes of the standing wave.

Figure 14 demonstrates this effect at $p_{0}=300, \Delta=-0.05$ and the same other conditions as before. We see that the momentum of the $|-\rangle$-component (the upper trajectory in this figure) oscillates in the range $(300,-300)$, and this component is trapped in the first well $(-\pi / 2 \leq x \leq \pi / 2)$. Whereas the $|+\rangle$-component moves in the positive direction splitting at each node. Estimates of the period of oscillations of the $|-\rangle$-component, $T \simeq 2240$, with the help of (23) and of the time when the centroid of the $|+\rangle$-component crosses the first node, $\tau_{1}^{(+)} \simeq 380$ (formula (29)), fit well the data in Fig. 14 .

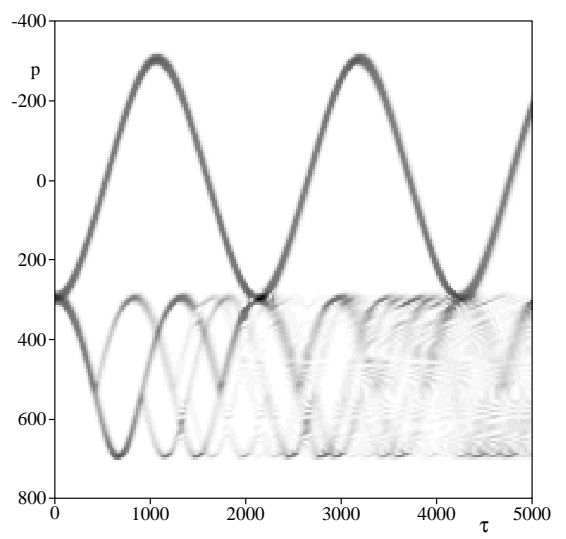

Fig. 14 Effect of simultaneous trapping of an atom in a well of the optical potential and its flight through the wave. The ground initial state, $\Delta=-0.05, p_{0}=300$. 


\section{Quantum-classical correspondence and manifestations of dynamical chaos in wave-packet atomic motion}

Dynamical chaos in classical systems is characterized by exponentially fast divergence of initially close trajectories in a bounded phase space. Such a behavior is possible because of the continuity of the classical phase space whose points (therefore, classical system's states) can be arbitrary close to each other. The trajectory concept is absent in quantum mechanics whose phase space is not continuous due to the Heisenberg uncertainty relation. The evolution of an isolated quantum system is unitary, and there can be no chaos in the sense of exponential sensitivity of its states to small variations in initial conditions. What is usually understand under "quantum chaos" is special features of the unitary evolution of a quantum system in the range of its parameter values and initial conditions at which its classical analogue is chaotic.

The question "what happens to classical motion in the quantum world" is a core of the problem of quantum-classical correspondence. In spite of years of discussions from the beginning of the quantum era, it is still unclear how classical features appear from the underlying quantum equations. It is especially difficult to specify what happens to classical dynamical chaos in the quantum world [31, 32, 33, 34, 35, 36, 37. The interest to the problem of "quantum chaos" is motivated by our desire to understand the quantum origin of the observed classical chaos.

In this section we establish a correspondence between the quantized motion of a two-level atom in a standing laser wave and its semiclassical analogue considered in the third section. Semiclassical equations (5) represent a nonlinear dynamical system with positive values of the maximal Lyapunov exponent in a wide range of the initial conditions and control parameters $\omega_{r}$ and $\Delta$. In other words, trajectories in the five-dimensional phase space are exponentially sensitive to small variations in initial conditions and/or parameters in those ranges. That local dynamical instability is a reason for chaotic Rabi oscillations and chaotic motion of the atomic center of mass discussed in the third section. In particular, it has been found that an atom is able to walk chaotically in a strictly periodic optical lattice without any noise or other random processes (see Fig. 2). The chaotic behavior is caused by jumps of the electric-dipole moment $u$ at the nodes of the standing wave (Fig. 3). It follows from Eqs. (5) that this quantity governs the atomic momentum. A stochastic map for the quantity $u$ (11) allowed to derive analytic expressions

for probability density functions of the atomic trapping and flight events that have been shown to fit well numerical simulation 23 .

It has been shown that sudden changes in the behavior of $u$ take place when we quantized the atomic motion (see Fig. 11) under the condition $\Delta \simeq \Delta_{0}$. Those changes are more smooth than the jumps of $u$ in the semiclassical case because a delocalized wave packet crosses a node for a finite time interval. 
The quantum analysis provides a clear reason for those jumps at $\Delta \simeq \Delta_{0}$, namely, it is nonadiabatic transitions between the quasienergy states $|+\rangle_{\Delta}$ and $|-\rangle_{\Delta}$ which occur when an atom crosses any node of the standing wave. Those jumps are accompanied by splitting of wave packets at the nodes. We may conclude that the proliferation of wave packets at the nodes of the standing wave is a manifestation of classical chaotic transport of an atom in an optical lattice that has been shown in Refs. 21, 24, 23. to take place in exactly the same ranges of initial conditions and control parameters. In particular, the effect of simultaneous trapping of an atom in a well of the optical potential and its flight in the same potential (Fig. 14) is a quantum analogue of a chaotic walking of an atom shown in Fig. 2 .

In conclusion we would like to discuss briefly the role of dissipation. We did not take into account any losses in our treatment. Coherent evolution of the atomic state in a near-resonant standing-wave laser field is interrupted by spontaneous emission events at random moments of times. The semiclassical Hamiltonian evolution between these events has been shown to be regular or chaotic depending on the values of the detuning $\Delta$ and the initial momentum $p_{0}$. We stress that dynamical chaos may happen without any noise and any modulation of the lattice parameters. It is a specific kind of dynamical instability in the fundamental interaction between the matter and radiation.

Dissipative transport of spontaneously emitting atoms in a 1D standingwave laser field has been studied in detail in Ref. 38, in the regimes where the underlying semiclassical Hamiltonian dynamics is regular and chaotic. A Monte Carlo stochastic wavefunction method was applied to simulate semiclassically the atomic dynamics with coupled internal and translational degrees of freedom. It has been shown in numerical experiments and confirmed analytically that chaotic atomic transport can take the form either of ballistic motion or a random walking with specific statistical properties. The character of spatial and momentum diffusion in the ballistic atomic transport was shown to change abruptly in the atom-laser detuning regime where the Hamiltonian dynamics is irregular in the sense of dynamical chaos. A clear correlation between the behavior of the momentum diffusion coefficient and Hamiltonian chaos probability has been found.

What one could expect if spontaneous emission would be taken into consideration with our fully quantum equations of motion? Any act of spontaneous emission interrupts a coherent evolution of an atom at a random time moment and is accompanied by a momentum recoil and a sudden transition of the atom into the ground state which is a superposition of the dressed states. The coherent evolution starts again after that. A collapse of the atomic wave function and a splitting of atomic wave packets are expected just after any spontaneous emission event. That additional splitting of wave packets at random time moments, besides of their proliferation at the nodes of a standing wave at $\Delta \simeq \Delta_{0}$, can improve the quantum-classical correspondence in the regime of Hamiltonian chaos. 
I thank L. Konkov and M. Uleysky for their help in preparing some figures. This work was supported by the Russian Foundation for Basic Research (project no. 09-02-00358) and by the Program "Fundamental Problems of Nonlinear Dynamics" of the Russian Academy of Sciences.

\section{References}

1. H. Haken, 1975, Analogy between higher instabilities in fluids and lasers, Phys. Lett. A, Vol. 53, 77-78.

2. P. I. Belobrov, G. M. Zaslavskii, G. Kh. Tartakovskii, 1976, Stochastic destruction of bound states in a system of atoms interacting with a radiation, Zh. Eksp. Teor. Fiz., Vol. 71, 1799-1812. [Sov. Phys. JETP, Vol. 44, 945-957].

3. R. M. Dicke, 1954, Coherence in spontaneous radiation, Phys. Rev., Vol. 93, 99-110.

4. E. T. Jaynes, F. W. Cummings, 1963, Comparison of quantum and semiclassical theories with application to the beam maser, Proc. IEEE, Vol. 51, 89-109.

5. W. K. Hensinger, H. Haffner, A. Browaeys, N. R. Heckenberg, K. Helmerson, C. McKenzie, G. J. Milburn, W. D. Phillips, S. L. Rolston, H. Rubinsztein-Dunlop and B. Upcroft, 2001, Dynamical tunnelling of ultracold atoms, Nature, Vol. 412, 52-55.

6. S. V. Prants, M. Edelman, and G. M. Zaslavsky, 2002, Chaos and flights in the atomphoton interaction in cavity QED, Phys. Rev. E, Vol. 66, art. 046222.

7. A.P. Kazantsev, G.I. Surdutovich, and V.P. Yakovlev, 1990, Mechanical Action of Light on Atoms, World Scientific, Singapore.

8. G. Grynberg and C. Robilliard, 2001, Cold atoms in dissipative optical lattices, Phys. Rep., Vol. 355, 335-451.

9. O. Morsch and M. Oberthaler, 2006, Dynamics of Bose-Einstein condensates in optical lattices, Rev. Mod. Phys., Vol. 78, 179-215.

10. F. L. Moore, J. C. Robinson, C. Bharucha, P. E. Williams, and M. G. Raizen, 1994, Observation of dynamical localization in atomic momentum transfer: a new testing ground for quantum chaos, Phys. Rev. Lett., Vol. 73, 2974-2977.

11. F. L. Moore, J. C. Robinson, C. F. Bharucha, B. Sundaram, M. G. Raizen, 1995, Atom optics realization of the quantum $\delta$-kicked rotor, Phys. Rev. Lett., Vol. 75, 4598-4601.

12. W. K. Hensinger, N.R. Heckenberg, G.J. Milburn, and H. Rubinsztein-Dunlop, 2003, Experimental tests of quantum nonlinear dynamics in atom optics, J. Opt. B: Quantum Semiclass. Opt., Vol. 5, R83-R120.

13. R. Graham, M. Schlautmann, and P. Zoller, 1992, Dynamical localization of atomicbeam deflection by a modulated standing light wave, Phys. Rev. A, Vol. 45, R19-RR22.

14. D. A. Steck, W.H. Oskay, and M. Raizen, 2001, Observation of chaos-assisted tunneling between islands of stability, Science, Vol. 293, 274-278.

15. S. Chu, L. Hollberg, J. E. Bjorkholm, A. Cable, and A. Ashkin, 1985, ThreeDimensional Viscous confinement and Cooling of Atoms by Resonance Radiation Pressure, Phys. Rev. Lett., Vol. 55, 48-51.

16. F. Bardou, J. P. Bouchaud, A. Aspect, and C. Cohen-Tannoudji, 2002, Lévy Statistics and Laser Cooling, Cambridge University Press, Cambridge.

17. S. Marksteiner, K. Ellinger, and P. Zoller, 1996, Anomalous diffusion and Levy walks in optical lattices, Phys. Rev. A, Vol. 53, 3409-3430.

18. S. V. Prants and V. Yu. Sirotkin, 2001, Effects of the Rabi oscillations on the atomic motion in a standing-wave cavity, Phys. Rev. A, Vol. 64, art. 033412.

19. S. V. Prants and L. E. Kon'kov, 2001, Chaotic motion of atom in the coherent field of a standing light wave, JETP Lett., Vol. 73, 180-183. [Pis'ma ZhETF, Vol. 73, 200-2004]. 
20. S. V. Prants, 2002, Chaos, fractals and flights of atoms in cavities, JETP Letters, Vol. 75, 651-658. [Pis'ma ZhETF, Vol. 75, 777-785].

21. V. Yu. Argonov and S. V. Prants, 2003, Fractals and chaotic scattering of atoms in the field of a stationary standing light wave, JETP, Vol. 96, 832-845. [Zh. Eksp. Teor. Fiz., Vol. 123, 946-961].

22. S. V. Prants and M. Yu. Uleysky, 2003, Atomic fractals in cavity quantum electrodynamics, Phys. Lett. A, Vol. 309, 357-362.

23. V.Yu. Argonov and S.V. Prants, 2007, Theory of chaotic atomic transport in an optical lattice, Phys. Rev. A, Vol. 75, art. 063428.

24. V. Yu. Argonov and S. V. Prants, 2006, Nonlinear coherent dynamics of an atom in an optical lattice, J. Russ. Laser Res., Vol. 27, 360-378.

25. S.V. Prants, M.Yu. Uleysky, and V.Yu. Argonov, 2006, Entanglement, fidelity, and quantum-classical correlations with an atom moving in a quantized cavity field, Phys. Rev. A, Vol. 73, art. 023807.

26. P. Gaspard, 1998, Chaos, Scattering and Statistical Mechanics, Cambridge University Press, Cambridge.

27. G. M. Zaslavsky, 2005, Hamiltonian Chaos and Fractional Dynamics, Oxford University Press, Oxford.

28. M. V. Budyansky, M. Yu. Uleysky, and S. V. Prants, 2004, Chaotic scattering, transport, and fractals in a simple hydrodynamic flow, JETP, Vol. 99, 1018-1027. [Zh. Eksp. Teor. Fiz., Vol. 126, 1167-1179].

29. A. P. Kazantsev, 1975, Recoil effect in a strong resoannt field, Sov. Phys. JETP, Vol. 40, 825-831. [1974, Zh. Eksp. Teor. Fiz., Vol. 67, 1660-1666].

30. P.E. Moskowitz, P.L. Gould, S.R. Atlas, and D.E. Pritchard, 1983, Diffraction of an atomic beam be standing-wave radiation, Phys. Rev. Lett., Vol. 51, 370-373.

31. G.P. Berman, G.M. Zaslavsky, 1978, Condition of stochasticity in quantum nonlinear systems, Physica A, Vol. 91, 450-460.

32. G. Casati, B.V. Chirikov, F.M. Izrailev, and J. Ford, 1979, Stochastic Behavior in Classical and Quantum Hamiltonian Systems, Lecture Notes in Physics, SpringerVerlag, Berlin.

33. G.M. Zaslavsky, 1981, Stochasticity in quantum systems, Phys. Rep., Vol. 80, 157-250.

34. M. Gutzwiller, 1990, Chaos in Classical and Quantum Mechanics, Springer-Verlag, New York.

35. L.E. Reichl, 1992, The Transition to Chaos in Conservative Classical Systems: Quantum Manifestations, Springer-Verlag, New York.

36. F. Haake, 1991, Quantum Signatures of Chaos, Springer-Verlag, Berlin. H.-J. Stöckmann, 1999, Quantum Chaos: An Introduction, Cambridge University Press.

37. D. Makarov, S. Prants, A. Virovlyansky, and G. Zaslavsky, 2010, Ray and Wave Chaos in Ocean Acoustics, World Scientific, Singapore.

38. V.Yu. Argonov and S.V. Prants, 2008, Theory of dissipative chaotic atomic transport in an optical lattice, Phys. Rev. A, Vol. 78, art. 043413. 